\title{
Practice Patterns Regarding Multidisciplinary Cancer Management and Suggestions for Further Refinement: Results from a National Survey in Korea
}

\author{
Yun-Gyoo Lee, MD ${ }^{1}$ \\ Sukjoong Oh, MD, $\mathrm{PhD}^{1}$ \\ Heejin Kimm, MD, PhD² \\ Dong-Hoe Koo, MD, PhD \\ Do Yeun Kim, MD, MPH, PhD ${ }^{3}$ \\ Bong-Seog Kim, MD, PhD \\ Seung-Sei Lee, MD, PhD ${ }^{1}$
}

\section{${ }^{1}$ Department of Internal Medicine,}

Kangbuk Samsung Hospital, Sungkyunkwan University School of Medicine, Seoul, ${ }^{2}$ Institute for Health Promotion and Department of Epidemiology and Health Promotion, Graduate School of Public Health, Yonsei University, Seoul, ${ }^{3}$ Department of Internal Medicine, Dongguk University Ilsan Hospital, Goyang, ${ }^{4}$ Department of Internal Medicine, Veterans

Health Service Medical Center, Seoul, Korea

Correspondence: Sukjoong Oh, MD, PhD Department of Internal Medicine,

Kangbuk Samsung Hospital, Sungkyunkwan University School of Medicine, 29 Saemunan-ro, Jongno-gu, Seoul 03181, Korea

Tel: 82-2-2001-2058

Fax: 82-2-2001-2088

E-mail: sukjoong.oh@samsung.com

Received October 27, 2016

Accepted February 5, 2017

Published Online February 22, 2017

\begin{abstract}
Purpose
This study was conducted to explore the process and operation of a cancer multidisciplinary team (MDT) after the reimbursement decision in Korea, and to identify ways to overcome the major barriers to effective and sustainable MDTs.
\end{abstract}

\begin{abstract}
Materials and Methods
Approximately 1,000 cancer specialists, including medical oncologists, surgical oncologists, radiation oncologists, pathologists, and radiologists in general hospitals in Korea were invited to complete the survey. The questionnaire covered the following topics: organizational structure of MDTs, candidates for consulting, the clinical decision-making initiative, and responsibility for dealing with legal disputes.
\end{abstract}

\section{Results}

We collected a total of 179 responses (18\%) from physicians at institutions where an MDT approach was active. A surgical oncologist (91\%), internist (90\%), radiologist (89\%), radiation oncologist (86\%), pathologist (71\%), and trainees (20\%) regularly participated in MDT operations. Approximately $55 \%$ of respondents stated that MDTs met regularly. In cases of a split opinion, the physician in charge (69\%) or chairperson (17\%) made the final decision, and most (86\%) stated they followed the final decision. About $15 \%$ and $32 \%$ of respondents were "very satisfied" and "satisfied," respectively, with the current MDT's operations. Among 38 institutional representatives, 34\% responded that the MDT operation became more active and $18 \%$ stated an MDT was newly implemented after the reimbursement decision.

\section{Conclusion}

The reimbursement decision invigorated MDT operations in almost half of eligible hospitals. Dissatisfaction regarding current MDTs was over $50 \%$, and the high discordance rates regarding risk sharing suggest that it is necessary to revise the current system of MDTs.

\section{Introduction}

To manage patients with advanced cancer in the most effective way, experts from different disciplines need to be engaged [1]. This need has resulted in introduction of the multidisciplinary team (MDT) approach, in which key professionals discuss a particular patient and contribute independently to clinical decisions [2]. Communication, coordination, and decision making between healthcare profes-
Key words

Reimbursement, Multidisciplinary, Korea sionals and patients have improved with the implementation of the MDT approach [3].

Because of these advantages, current clinical guidelines recommend discussing the diagnostic and/or therapeutic plan with an MDT for localized or locally advanced nonsmall cell lung cancer [4], synchronous metastatic/first recurrent metachronous metastatic colon cancer [5], locoregional stomach cancer [6], and most types of head and neck cancer [7]. Although the concepts of the MDT approach are considered good clinical practice in oncology, MDT organi- 
zation and implementation take a substantial amount of time and additional efforts by the team's relevant healthcare professionals. In addition, there are practical barriers to active and sustainable implementation of an MDT [3].

To improve patient-centered cancer care by invigorating the MDT approach and to enhance the benefits coverage in recompense for abrogation of the physician surcharge, the Korean Health Authority decided to reimburse the costs of the "Multidisciplinary Care Service" on August 1, 2014. The Korean model of the MDT is provided for patients in superior general hospitals or the Korea cancer center hospitals who have a clinically suspicious or pathologically confirmed malignancy. The MDT includes four or more-expert services and is offered in the outpatient clinic as a face-to-face service between patient and physicians. The hospital can charge 113,210 won (102 US dollars) for the 4-expert service or 141,510 Korean won (128 US dollars) for the 5- or moreexpert service up to three times per primary tumor.

This study was conducted to explore the process and operation of cancer MDTs after the reimbursement decision was made in Korea, and to identify ways to overcome the major barriers to effective and sustainable MDTs.

\section{Materials and Methods}

\section{Development of the survey}

Survey Monkey (https://ko.surveymonkey.com) was used to create a web-based online survey. The survey consisted of 25 questions about the structure, extent, and functioning of MDTs in Korea. A follow-up questionnaire consisted of four items concerning changes in practice after the reimbursement decision went into effect.

\section{Participants}

To complete the survey, we invited approximately 1,000 members of the Korean Association for Clinical Oncology, Korean Society of Surgical Oncology, Korean Society for Radiation Oncology, Korean Society of Pathologists, and Korean Society of Radiology including medical oncologists, surgical oncologists, radiation oncologists, pathologists, and radiologists, as well as trainees, nurses, and clinical research coordinators. The follow-up survey was only sent to representative medical oncologists in each hospital as part of the Survey of Medical Oncology Status in Korea (SOMOS-K) [8].

From October 2014 to January 2015, e-mail invitations including a link to the survey were sent to participants after approval from the relevant society. Repeated invitations after the initial distribution were sent to non-responders 8 weeks and again 12 weeks later. Responses were automatically collected by the online server.

\section{Statistical analysis}

"Respondent" refers to an attending physician who is the institutional representative that completed the questionnaire. Fisher exact test with a significance level of $<0.05$ was used to compare differences in binary variables. All statistical analyses were performed using the Stata ver. 13.0 software (Stata Corp LP, College Station, TX).

\section{Results}

\section{Responses}

Included in the study were 179 responses from physicians at institutions where an MDT approach was active (Table 1). Of these 179 respondents, 70 (39\%) were internists (58 medical oncologists, 12 pulmonologists), 23 (13\%) surgeons, 22 $(12 \%)$ radiation oncologists, $41(23 \%)$ radiologists, and 23 $(13 \%)$ pathologists. A total of 38 institutional representatives responded to a follow-up questionnaire.

\section{Structure and functioning of MDTs}

The MDT meetings were regularly attended by surgical oncologist (91\%), an internist including a medical oncologist and pulmonologists $(90 \%)$, radiologist $(89 \%)$, radiation oncologist $(86 \%)$, pathologist $(71 \%)$, and trainees including residents or fellows (20\%) (Table 2). A total of $86 \%$ of the MDTs had a designated $(56 \%)$ or rotated $(30 \%)$ chairperson. Overall, 55\% of the respondents stated that their MDTs met regularly, 30\% every week, $19 \%$ more than once a week, $4 \%$ every 2 weeks, and $1 \%$ every month.

A total of $60 \%$ of MDTs discussed cancer patients in all stages, and the majority (98\%) of MDTs meetings were held to determine the therapeutic plan after the pathological diagnosis.

\section{Clinical decision making}

In case of a split opinion, the physician in charge $(69 \%)$ or chairperson $(17 \%)$ made the final decision and there was a majority vote $(15 \%)$ (Table 3$)$. About $90 \%$ of respondents indicated that it was not mandatory for the treating physicians to implement the final recommendation of the MDTs; however, most $(86 \%)$ indicated that they did follow the rec- 
Table 1. Characteristics of the 179 respondents with an MDT at their center

\begin{tabular}{|c|c|}
\hline Characteristic & No. $(\%)$ \\
\hline \multicolumn{2}{|l|}{ Age group (yr) } \\
\hline $30-40$ & $49(27)$ \\
\hline $40-50$ & $78(44)$ \\
\hline$>50$ & $52(29)$ \\
\hline \multicolumn{2}{|l|}{ Clinical career (yr) } \\
\hline$<5$ & $24(13)$ \\
\hline $5-10$ & $49(27)$ \\
\hline $10-20$ & $60(34)$ \\
\hline$>20$ & $46(26)$ \\
\hline \multicolumn{2}{|l|}{ Department } \\
\hline Internal Medicine & $70(39)$ \\
\hline Medical Oncology & $58(32)$ \\
\hline Pulmonology & $12(7)$ \\
\hline General Surgery & $23(13)$ \\
\hline Radiation Oncology & $22(12)$ \\
\hline Radiology & $41(23)$ \\
\hline Pathology & $23(13)$ \\
\hline \multicolumn{2}{|l|}{ Specialty (multiple) } \\
\hline Stomach & $59(33)$ \\
\hline Colon & $60(34)$ \\
\hline Lung & $78(44)$ \\
\hline Breast & $51(28)$ \\
\hline Hepato-biliary & $56(31)$ \\
\hline Genitourinary & $40(22)$ \\
\hline Head and neck & $49(27)$ \\
\hline \multirow[t]{2}{*}{ Rare malignancy } & $36(20)$ \\
\hline & $179(100)$ \\
\hline
\end{tabular}

MDT, multidisciplinary team.

ommendation. Regarding the hypothetical question of a lawsuit being brought by a patient who underwent treatment according to the decision of the MDT, $37 \%$ of respondents stated that the treating physicians are responsible, while 38\% replied that all members of the MDT should share the responsibilities. In terms of the aforementioned risk-sharing issues, experts from different disciplines had significantly different opinions ( $p=0.039$ between groups). Specifically, internists, surgeons, and radiation oncologists were more likely to share their responsibility, while radiologists and pathologists were less likely to accept responsibility for the decision.

\section{Satisfaction}

About 15\% and 32\% of respondents were "very satisfied" and "satisfied," respectively, with how the current MDTs
Table 2. Structure of the MDT for cancer care in Korea

Structure Percentage ${ }^{a)}$

\begin{tabular}{lc}
\hline Participants & \\
\hline Internal Medicine & 90 \\
\hline Radiology & 89 \\
\hline General Surgery & 91 \\
\hline Radiation Oncology & 86 \\
\hline Pathology & 71 \\
\hline Trainees (residents or fellows) & 20 \\
\hline Chairperson & \\
\hline No & 14 \\
\hline Yes & - \\
\hline Designated & 56 \\
\hline Rotating & 30 \\
\hline Schedule & \\
\hline Irregular meeting (in case) & 45 \\
\hline Regular meeting & 55 \\
\hline$\quad$ More than one meeting a week & 19 \\
\hline$\quad$ Every week & 30 \\
\hline Every 2 weeks & 4 \\
\hline Every month & 1 \\
\hline Stage in discussion & \\
\hline All stages & 60 \\
\hline Localized stage & 39 \\
\hline Recurrent or metastatic stage & 27 \\
\hline Reasons for an MDT & 2 \\
\hline To diagnose cancer & 98 \\
\hline To decide therapeutic plan after diagnosis \\
\hline To decide palliative care plan after recurrence \\
\hline
\end{tabular}

${ }^{a)}$ We calculated the proportion of answers based on 70 multidisciplinary teams (MDTs) of 179 respondents.

functioned. Regarding the degree of satisfaction among experts from different disciplines, there was no significant difference between experts ( $p=0.196$ ). In particular, internists and surgeons were more satisfied with MDTs than other doctors were ( $\mathrm{p}=0.027$ ) (Fig. 1).

\section{Changes after the reimbursement decision}

Among 38 institutional representatives, 34\% (13/38) responded that MDT operations became more active and $18 \%$ (7/38) stated that an MDT was newly implemented after the reimbursement decision.

\section{Barriers to improvements}

More than half of respondents (56\%) replied that the current costs of reimbursement should be raised (the current 
Table 3. Responses to various questions in the survey, especially pertaining to clinical decision making

\begin{tabular}{|c|c|}
\hline Question & Percentage ${ }^{a)}$ \\
\hline \multicolumn{2}{|l|}{$\begin{array}{l}\text { Who makes the final decision/ } \\
\text { recommendation in case there } \\
\text { is more than one opinion? }\end{array}$} \\
\hline Physician in charge & 69 \\
\hline Chairperson & 17 \\
\hline Majority vote & 15 \\
\hline \multicolumn{2}{|l|}{$\begin{array}{l}\text { Is it mandatory for the treating doctor } \\
\text { to implement the decision/ } \\
\text { recommendation of the MDT? }\end{array}$} \\
\hline Yes & 8 \\
\hline $\begin{array}{l}\text { No, but follow the decision/ } \\
\text { recommendation }\end{array}$ & 86 \\
\hline $\begin{array}{l}\text { No, decide regardless of } \\
\text { the decision/recommendation }\end{array}$ & 6 \\
\hline \multicolumn{2}{|l|}{$\begin{array}{l}\text { In case of a lawsuit brought on by } \\
\text { a patient who underwent treatment } \\
\text { according to the decision of the MDT, } \\
\text { who do you think is/are } \\
\text { legally responsible? }\end{array}$} \\
\hline All members of the MDT & 38 \\
\hline Treating doctor & 37 \\
\hline Hospital & 18 \\
\hline Chairperson & 3 \\
\hline Others & 3 \\
\hline
\end{tabular}

a) We calculated the proportion of answers based on 70 multidisciplinary teams (MDTs) of 179 respondents. cost is 113,210 Korean won/102 US dollar for 4-expert services, and 141,510 won/128 US dollar for 5- or more-expert services). About $42 \%$ insisted that multidisciplinary care service should be expanded to include inpatient MDTs. Moreover, 30\% demanded that the option of face-to-face meetings should be eliminated and the number of experts who participate in MDTs should be regulated. Finally, 12\% of respondents noted that prerequisites regarding the institutions allowed to have an MDT should be relaxed from superior general hospital to general hospitals.

\section{Discussion}

We found that the reimbursement decision made by government health authorities invigorated MDT operations in almost half of eligible hospitals, suggesting that the reimbursement decision could affect our practice considerably. Moreover, dissatisfaction of over 50\% regarding current MDTs and high discordance rates regarding risk sharing were observed. Given the absence of specific guidance for MDTs, the implementation and organizational structure were variable across institutions.

The reimbursement to the "Multidisciplinary Care Service" has changed the practice patterns of healthcare professionals in Korea considerably. Approximately one third of institutional representatives stated that the MDT operation became more active and 18\% responded that an MDT was newly implemented. Despite these changes, a few important issues concerning regulations remain. Specifically, $56 \%$ of respondents stated that the cost of MDTs is not sufficient.

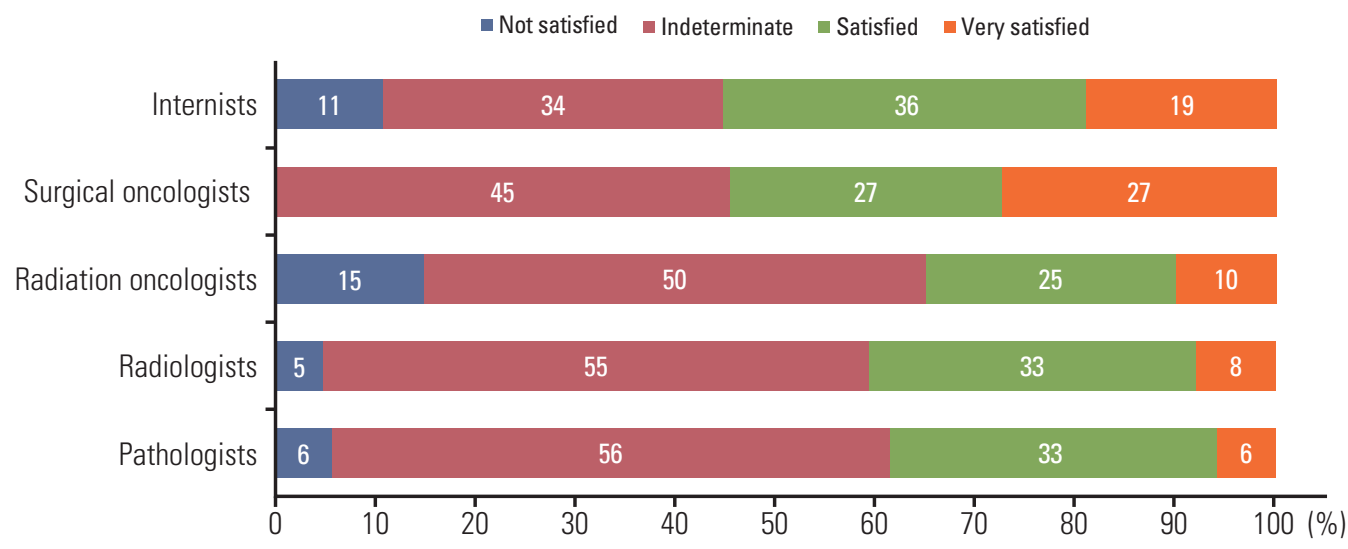

Fig. 1. Satisfaction with how the current multidisciplinary team functioned. $p=0.196$ between groups. $p=0.027$ between internists/surgical oncologists versus radiation oncologists/radiologists/pathologists. 
Currently, the MDT operation costs 102 US dollars for 4-expert service, which is approximately 25 US dollars per individual expert's consultation. In cases in which participation in MDT requires considerable time and extra effort (in our survey, more than half of respondents noted that), the current cost of reimbursement should be raised. Additionally, $42 \%$ of respondents stated that a restriction to outpatient consultation in MDT is not rational. According to the rule of outpatient consultation, the hospital cannot charge a service fee for inpatient MDT consultation. Our respondents were concerned that restriction to outpatient consultation will eventually discourage the experts' willingness to participate in MDT. Additionally, the current regulations do not accept the non-face-to-face form of MDT, including discussions in conference and tumor boards. Finally, $12 \%$ of respondents expressed concern that the prerequisites regarding eligible institutions (reimbursement for only 43 superior general hospitals or one Korean cancer center hospital) could limit the chance to join MDTs by patients as well as healthcare professionals. These barriers expressed by healthcare providers might limit the expansion of MDTs and cause perfunctory meetings to be held only to collect payments. Therefore, standard guidelines and the establishment of a flexible MDT model are necessary.

MDTs could improve clinical outcomes and patient satisfaction by shortening the time to diagnosis and therapeutic intervention, improving adherence to guidelines, and greater inclusion in clinical trials [9-11]. However, evidence for survival benefit and efficiency are not strong $[3,12,13]$. Therefore, future investigations regarding the effectiveness of MDT operations in Korea are warranted. Additionally, participation in a discussion between experts from different disciplines will help improve physicians' practical knowledge beyond theoretical information. Our survey showed that only $20 \%$ of MDT meetings invited residents or fellows. Therefore, we actively recommend that our trainees partici- pate in MDTs.

Some limitations must be noted. First, our study had a low response rate. This was primarily because we did not send our questionnaire to active members with current clinical activities, but rather to all members of corresponding academic societies, which include faculties, trainees, nurses, and clinical research coordinators. To overcome this limitation, we sent a follow-up questionnaire concerning changes in practice after reimbursement decision only to representative medical oncologists in each hospital. Second, the retrospective nature of our survey is subject to recall bias of all respondents. Because there is a paucity of data regarding MDT before the reimbursement decision, we directly asked our respondents about the actual changes in clinical practice between pre and post reimbursement, which might have caused recall bias.

The start of the "Multidisciplinary Care Service" is an important step in optimal cancer care in the Korean health system. The unceasing efforts to establish the best model and the proper rewards are necessary to expand MDTs across the country. Moreover, investigation of the outcome after the reimbursement decision is warranted to revise regulations and influence the regulatory body.

\section{Conflicts of Interest}

Conflict of interest relevant to this article was not reported.

\section{Acknowledgments}

This study was supported by a 2014 Academic Awards grant from the Korean Association for Clinical Oncology (KACO) and presented in part at the 2015 Annual Meeting of the KACO. The authors express our appreciation to all doctors for participation in the questionnaire and their valuable support.

\section{References}

1. Gouveia J, Coleman MP, Haward R, Zanetti R, Hakama M, Borras JM, et al. Improving cancer control in the European Union: conclusions from the Lisbon round-table under the Portuguese EU Presidency, 2007. Eur J Cancer. 2008;44: 1457-62.

2. European Partnership Action Against Cancer Consensus Group, Borras JM, Albreht T, Audisio R, Briers E, Casali P, et al. Policy statement on multidisciplinary cancer care. Eur J Cancer. 2014;50:475-80.

3. Fleissig A, Jenkins V, Catt S, Fallowfield L. Multidisciplinary teams in cancer care: are they effective in the UK? Lancet
Oncol. 2006;7:935-43.

4. Ettinger DS, Wood DE, Akerley W, Bazhenova LA, Borghaei $\mathrm{H}$, Camidge DR, et al. NCCN guidelines insights: non-small cell lung cancer, version 4.2016. J Natl Compr Canc Netw. 2016;14:255-64.

5. Benson AB 3rd, Venook AP, Bekaii-Saab T, Chan E, Chen YJ, Cooper HS, et al. Colon cancer, version 3.2014. J Natl Compr Canc Netw. 2014;12:1028-59.

6. Ajani JA, D'Amico TA, Almhanna K, Bentrem DJ, Besh S, Chao J, et al. Esophageal and esophagogastric junction cancers, version 1.2015. J Natl Compr Canc Netw. 2015;13:194-227. 
7. Pfister DG, Spencer S, Brizel DM, Burtness B, Busse PM, Caudell JJ, et al. Head and neck cancers, version 1.2015. J Natl Compr Canc Netw. 2015;13:847-55.

8. Kim DY, Lee YG, Kim BS. Survey of Medical Oncology Status in Korea (SOMOS-K): a national survey of medical oncologists in the Korean Association for Clinical Oncology (KACO). Cancer Res Treat. 2017;49:588-94.

9. Pawlik TM, Laheru D, Hruban RH, Coleman J, Wolfgang CL, Campbell K, et al. Evaluating the impact of a single-day multidisciplinary clinic on the management of pancreatic cancer. Ann Surg Oncol. 2008;15:2081-8.

10. Bjegovich-Weidman M, Haid M, Kumar S, Huibregtse C, McDonald J, Krishnan S. Establishing a community-based lung cancer multidisciplinary clinic as part of a large integrated health care system: aurora health care. J Oncol Pract. 2010;6:e27-30.

11. Hammond DB. Multidisciplinary cancer care in a community hospital setting: challenges and rewards. J Oncol Pract. 2010;6: 281-3.

12. Coory M, Gkolia P, Yang IA, Bowman RV, Fong KM. Systematic review of multidisciplinary teams in the management of lung cancer. Lung Cancer. 2008;60:14-21.

13. Kesson EM, Allardice GM, George WD, Burns HJ, Morrison DS. Effects of multidisciplinary team working on breast cancer survival: retrospective, comparative, interventional cohort study of 13722 women. BMJ. 2012;344:e2718. 